\title{
ROYAL SOCIETY \\ OF CHEMISTRY
}

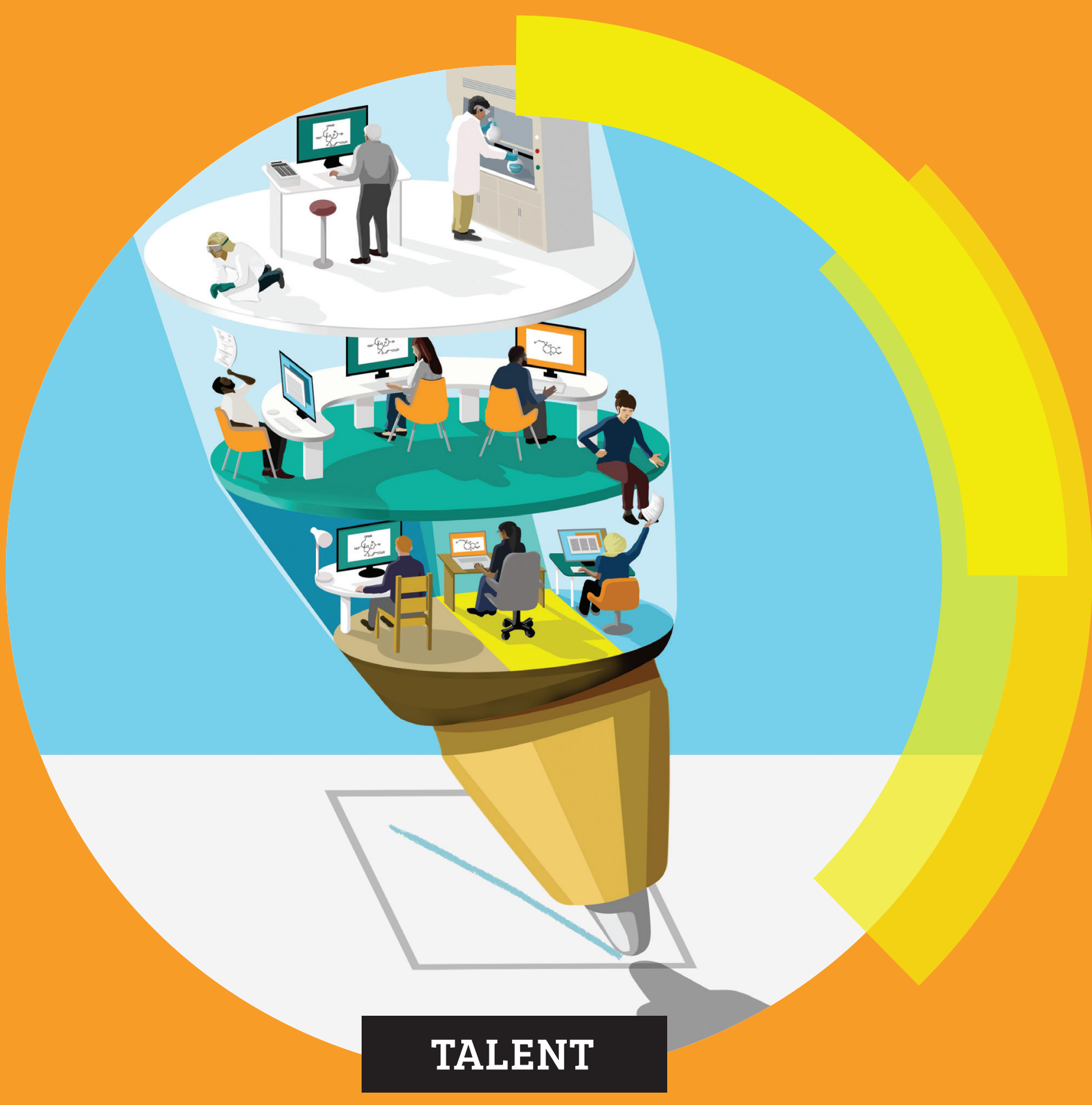

\section{Is publishing in the chemical sciences gender biased?}

Driving change in research culture 
1. Foreword

2. Executive summary

3. Background to this study

4. Methodology

5. Gender balance in our publishing community

6. What are the gender patterns of manuscript submissions?

7. How does gender affect manuscript review and acceptance rates?

8. Are female authors cited less?

9. Conclusion and recommendations

10. What we will do

11. References and acknowledgments
IS PUBLISHING IN THE CHEMICAL SCIENCES GENDER BIASED?

1.

\section{Foreword}


Chemistry should be for everyone. For the chemical sciences to prosper and deliver against global challenges, we must attract, develop and retain a diverse range of talented people. That's not merely an opinion - diverse teams deliver better results.

As a professional and membership body and a leading voice for the chemistry community, we have a responsibility to promote inclusivity and accessibility in order to improve diversity. When we audited the diversity landscape of the chemical sciences, we uncovered a lack of data and a need for greater transparency.

That is why we undertook this study to assess gender bias in our publishing activities. We are a global not-for-profit chemical science publisher reinvesting any surplus we make back into the chemistry community. Analysing and making data available from our own publishing, supports the community by bringing to light the hidden inclusion challenges that need tackling.

We found that there is a complex interaction of subtle biases occurring throughout the publishing pipeline, which combine to put women at a disadvantage when disseminating their research. We must recognise where this happens. We are committed to further scrutinising our own processes at each stage - and we are calling on other publishers to do the same. We want to work together to make scientific peer-reviewed publishing fit for the modern age.

Ensuring that the chemical science community fairly encourages, enables equal access and retains a more diverse range of voices will lead to better science and, by extension, will benefit society.

That's why we are working to make chemistry for everyone.

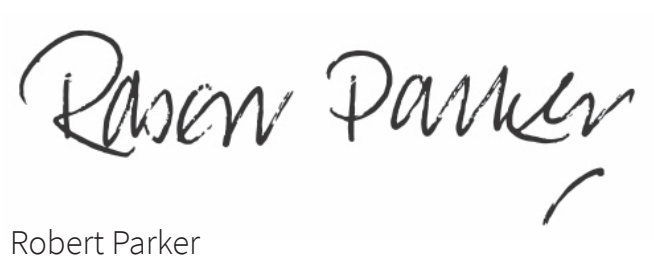

Chief Executive, Royal Society of Chemistry
2.

\section{Executive summary}


In early 2018, we published a report, Diversity andscape of the Chemical Sciences ${ }^{1}$, which raised many questions about the current state of diversity the chemical sciences and in particular highlighted the lack of progression of women. We explored the causes of poor retention and progression of women in our Breaking the Barriers ${ }^{2}$ report and made commitments to counteract them.

We are not only a professional body but also a publisher of peer reviewed research articles for the chemical sciences. Recognising that both the publication of research articles and the number of citations that those articles gather remain established markers of scientific success, we have carried out the first in-depth gender analysis of each stage of the publication process within the chemical sciences community.
Analyses of the gender profile of international publishing outputs, authorship and peer review ${ }^{46}$ have highilighted that both gender and geography have an impact on article acceptance and citation rates.

Here*, we have analysed the gender profile of the publishing pipeline of the Royal Society of Chemistry's journals between 2014 and 2018, showing that there are biases at each stage in the publication process. This report also contains views from the chemical science research community about the biases within publishing, the factors that might be contributing to these biases, and what we can do to tackle them.

It is likely that many of these biases are inadvertent; however beyond the response from our interviewees does not make assumptions of biases are introduced, consciously or unconsciously.

Biases exist at each step of the publishing profile. Many of these biases appear minor in isolation, yet their combined effect puts women at a significant disadvantage. WDomen are less likely to hold positions towards the end of the author list, in particular that

Women are less likely than men to submit to journals with higher impact factors, and they are also more likely to have an article rejected without review.

Women are under-represented as reviewers but are more likely to be chosen to review articles by female corresponding authors.

目 Both reviewer gender and author gender affects article acceptance.

몰 Biases operate at editorial level too. The choice of reviewer and editorial agreement with a review are influenced by gender.

Women cite fewer research papers than men overall, and men are less likely than women to cite papers authored by women.

\section{What are the consequences for female authors?}

Our findings show that biases do exist both pre- and post-publication

These subtle biases, which occur throughout the publishing pipeline, combine to put women at a disadvantage when disseminating their research. Female authors are less likely to benefit from the visibility provided by being a corresponding author

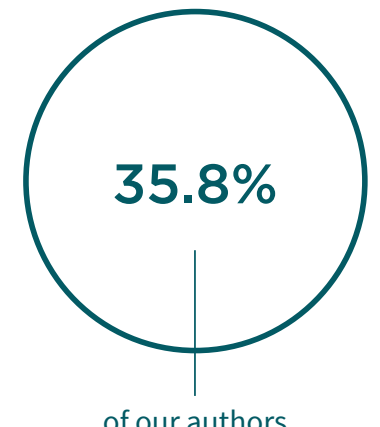

of our authors are women

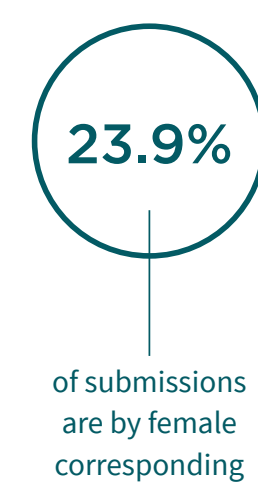

corresponding

authors

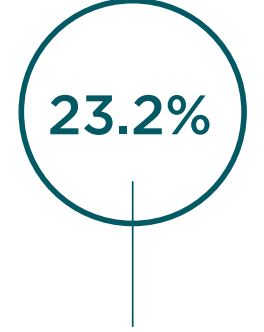

of papers accepted by editors and sen for peer review are by female corpond

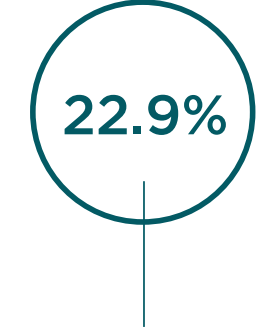

of papers accepted of citations have for publication acorresponding after peer review author who is a are by female woman authors

\section{What can we do about it?}

Only by recognising the biases that are introduced at decision points by authors, reviewers, editors and publishers, can we act to reduce them.

We will therefore continue to scrutinise our own processes at each stage of the publishing pipeline to aim to eliminate these sources of bias.

\section{We have identified four key areas for action. We will:}

1 Increase transparency

Undertake comprehensive analysis and reporting of our authors, reviewers and editorial decision makers by sub-discipline - and publish this annually. We call on other publishers to do the same.

\section{Empower and innovate}

Provide new training and resources to empower our editors to eliminate bias. We will test new models throughout the publishing profile to address bias from submission to publication
2 Reflect our research community Recruit and train reviewers, editorial board members and associate editors to reflect the current gender balance of our research community: our target for 2022 is at least $36 \%$ women.

\section{Encourage intervention}

Partner with others and lead the development of a new Inclusion \& Diversity Framework for Action to set the standard for driving change within the academic publishing industry. 
As a leading voice and publisher for the chemistry community, we have an opportunity to promote inclusivity and accessibility in order to improve diversity within the chemical sciences. This is not only a moral obligation - by increasing the diversity of voices within chemistry we can increase the quality of research, inspire and attract the next generation of chemists, and ensure that chemistry is relevant to al in society.

\section{Background to this study}

Our 2018 report, Diversity Landscape of the Chemical

Sciences ${ }^{1}$, highlighted the need for more progress

around gender equality, especially regarding the

leadership positions.

Other organisations have previously identified gender biases specifically within publishing.

Elsevier's Gender in the Global Research Landscape analysis ${ }^{4}$ examined the outputs, quality and impact of STEMM research worldwide by gender, and reported several findings relevant to our current study:
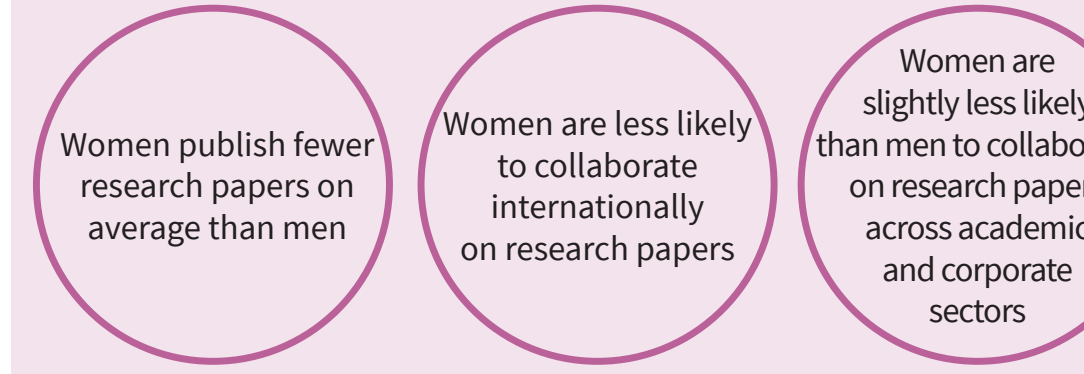

slightly less likely than men to collaborate on research papers across academic and corporate

sectors

In 2018 , the Institute of Physics (IOP) published an analysis of

In 2018, the Institute of Physics (IOP) published an analysis of
inclusion and diversity within peer review at IOP Publishing using gender and geographical data on authors, reviewers and editorial board members between 2014 and 2018. It was found that:

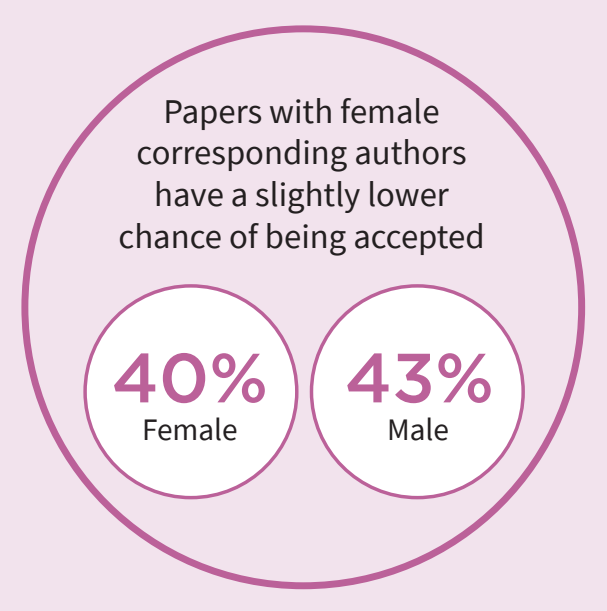

An examination
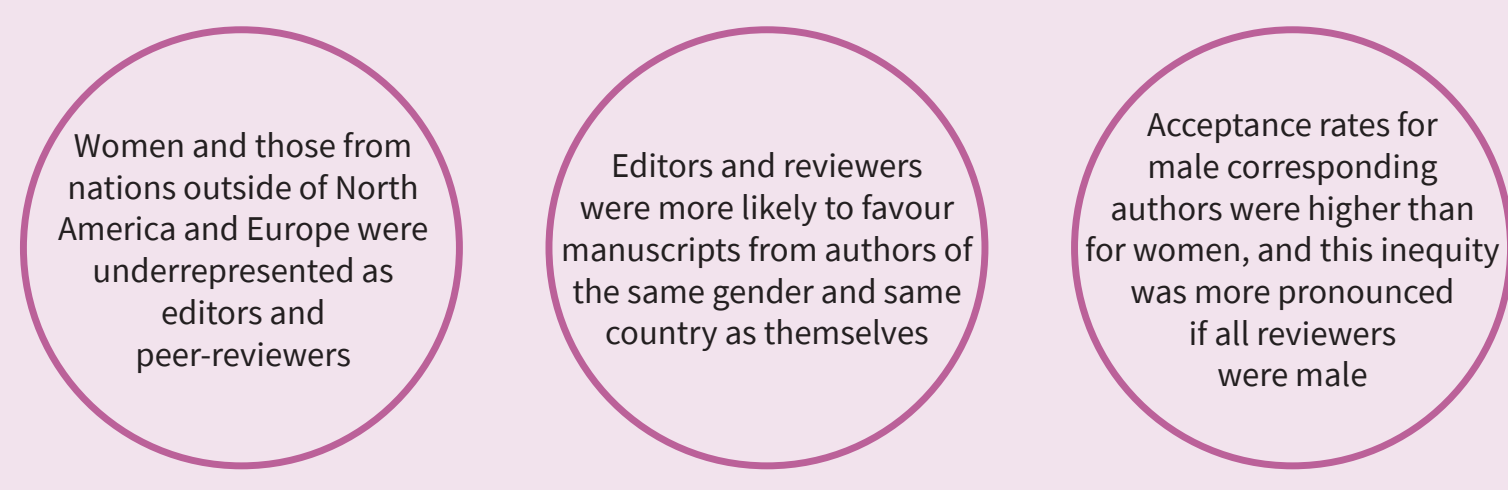
Publication metrics remain well recognised markers of scientific success and have an impact on career progression and therefore retention. Any biases within the publishing system, added to the barriers we previously uncovered ${ }^{2}$, have the potential to culminate in women having an undeservedly poorer publication record

That is why we have carried out the first in-depth gender analysis of each stage of the publication pipeline within the chemical science community.

"The knock-on effect is not simply that much fantastic research never sees the light of day. It is also that many talented people from minority backgrounds do not see their careers progress in the way their excellence would warrant. This is not good for science, let alone the individuals."

Melinda Duer and Dame Athene Donald Times Higher Education supplement 2019

Our publishing activity

The Royal Society of Chemistry publishes more than 40 peer-reviewed journals (over 35,000 articles in 2018), around 2,000 book titles and a collection of online databases and literature updating services. Our international publishing portfolio covers the core chemical sciences including related fields such as biology, biophysics, energy and environment, engineering, materials, medicine and physics.

An overview of our publishing process:

4.

\section{Methodology}

to peer review to publication, every paper submitted to our journals will go through the publishing process shown below. The editorial structure of the different journals, however, does vary slightly. Some have associate editors, active researchers in the field, who handle papers and work closely with the in-house editorial team. Others hav teams of in-house specialist editors. All editorial teams are fellow scientists who work closely with our international authors, reviewers and readers throughout the publishing process.

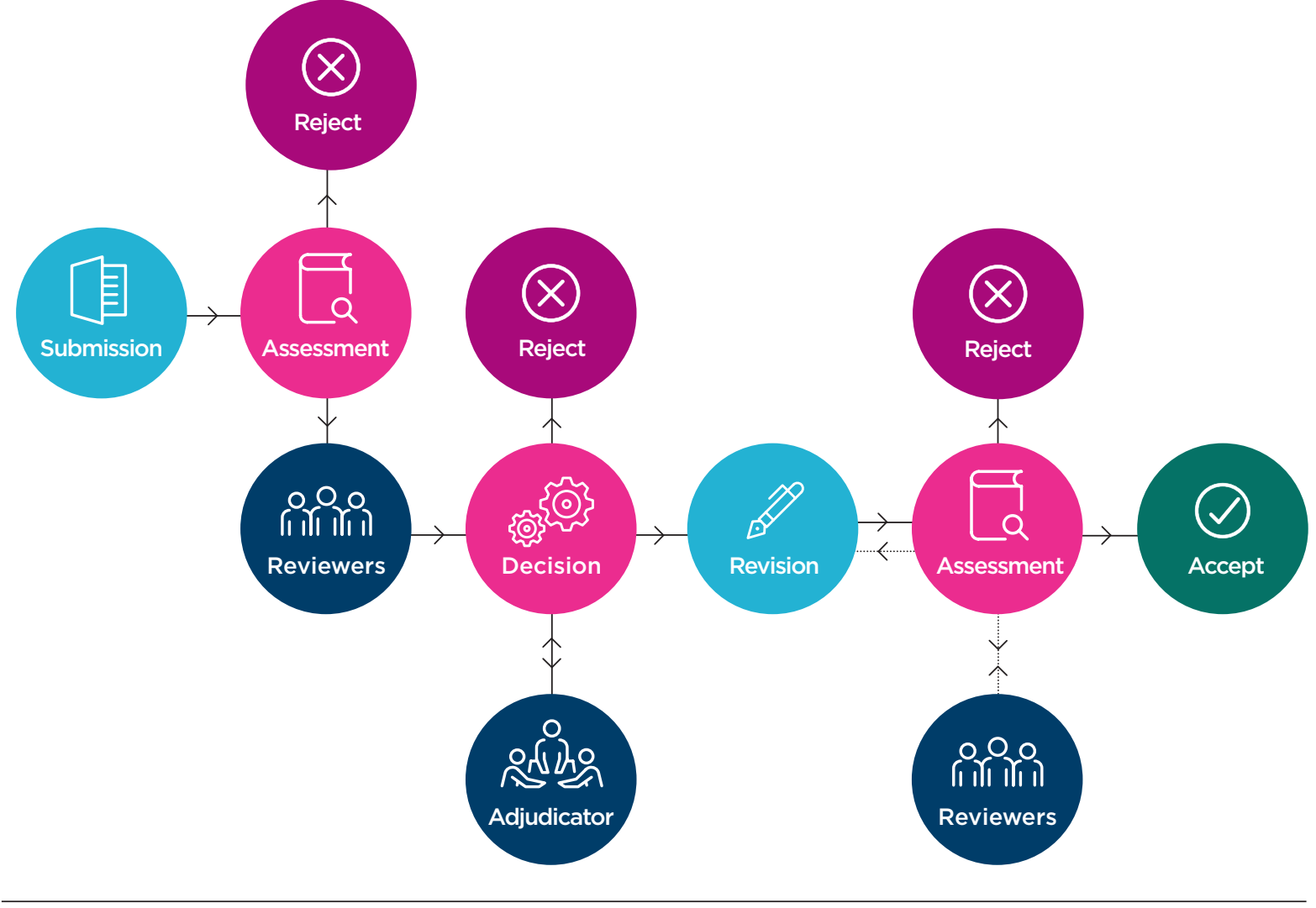


In this study, we analysed the gender profile of authors of 717,108 manuscript submissions across all our journals from January 2014 until the end of July 2018 and 141,073 citations between our journals Irom August 2011 until September 2018. Gender was assigned to names by following the approach (en Profiles in UK Patenting repoit

We used these data to:

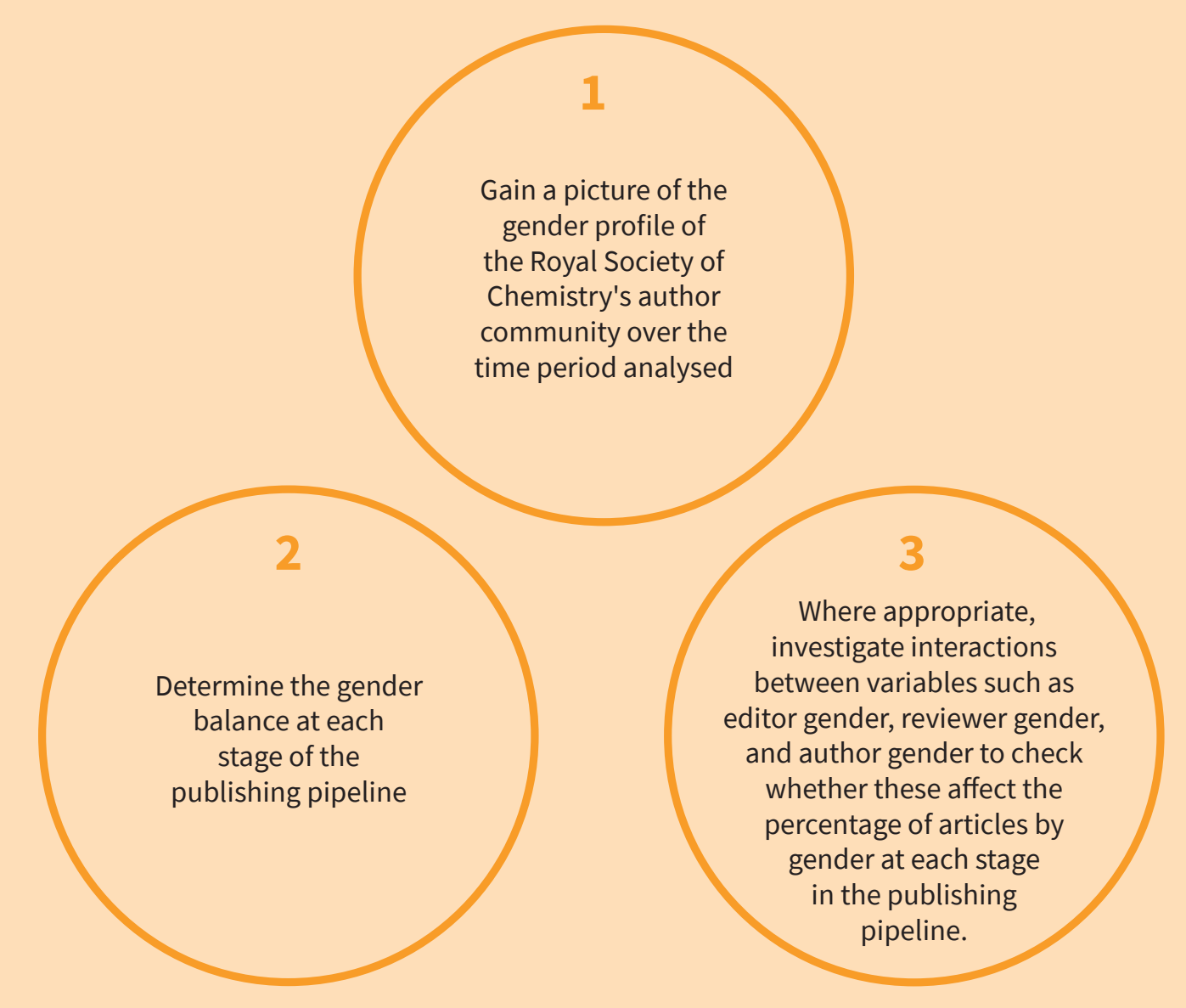

The results that follow have been compared to the baseline of female chemistry researchers in the Royal Society of Chemistry's author community.
On 政 percentage of thany terms. Calculations reflect the that the data set?
5.

\section{Gender balance in our publishing} community 
We investigated the gender balance among Wifferent publishing roles, to uncover how women are represented at various stages of the publishing pipeline.

The proportion of female authors submitting to our journals was $35.8 \%$. This number tallies with
other measures we used to assess the overall gender bus

Compared to this baseline we found that there were:

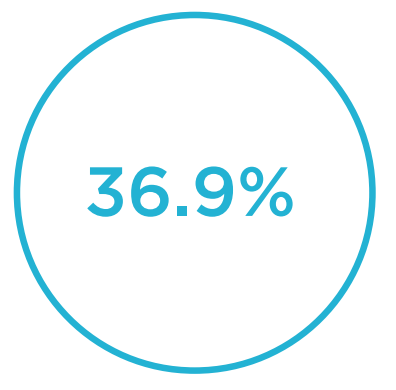

Slightly more female first authors

Substantially fewer female corresponding

authors

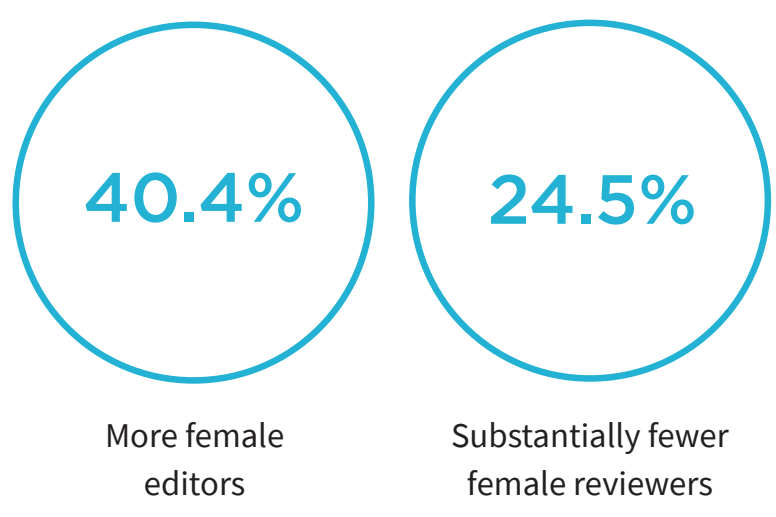

The data regarding female corresponding authors are interesting in light of previous data from our own analyses ${ }^{1}$ which show that female chemistry researchers are not progressing to senior positions Does this potopotion as their male counterparts. Does his potential lack of visibility as corresponding uthors impact on women's academic progression? Another possible impediment to women's progression is that they are less likely to collaborate internationally when publishing research articles. Ascertaining if there are barriers that prevent women from leading international collaborations is outside the scope of this work and something to be explored in the future.

The low proportion of female peer reviewers seen across our journals is mirrored by data published in the physics community. The IOP found no significan difference in the propensity for men or women to chept review invitations, which suggests that there is a pool of women who are not being inviced to review. provided by women is principally due to them being provided by women is principaly due to then being

"...when I choose reviewers as an editor I certainly try to ask as many women as men.... can see how this happens because people talk a lot about asking the 'very important person'.. I actually know what 'big people' means... mostly one type of person. 9

Author, reviewer and edito
What is the proportion of women in the RSC publishing community?

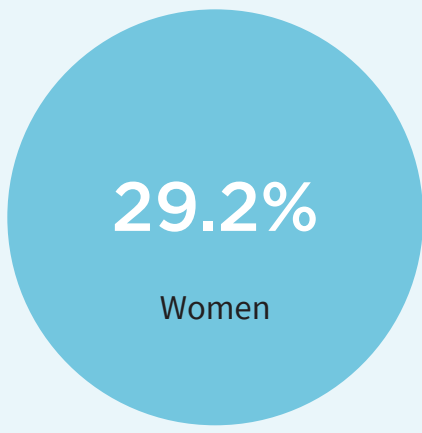

Corresponding authors

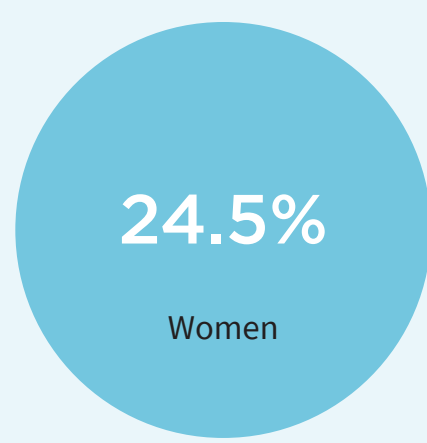

All reviewers

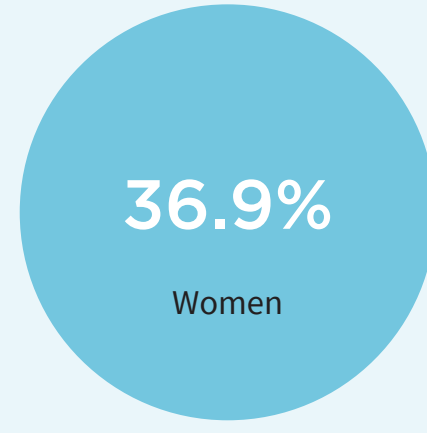

First authors

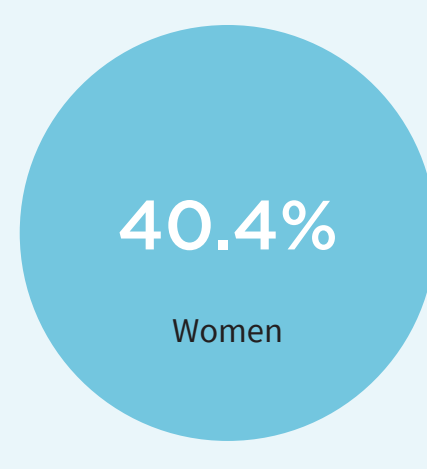

All editors 
6.

\section{What are the gender patterns of} manuscript submissions?

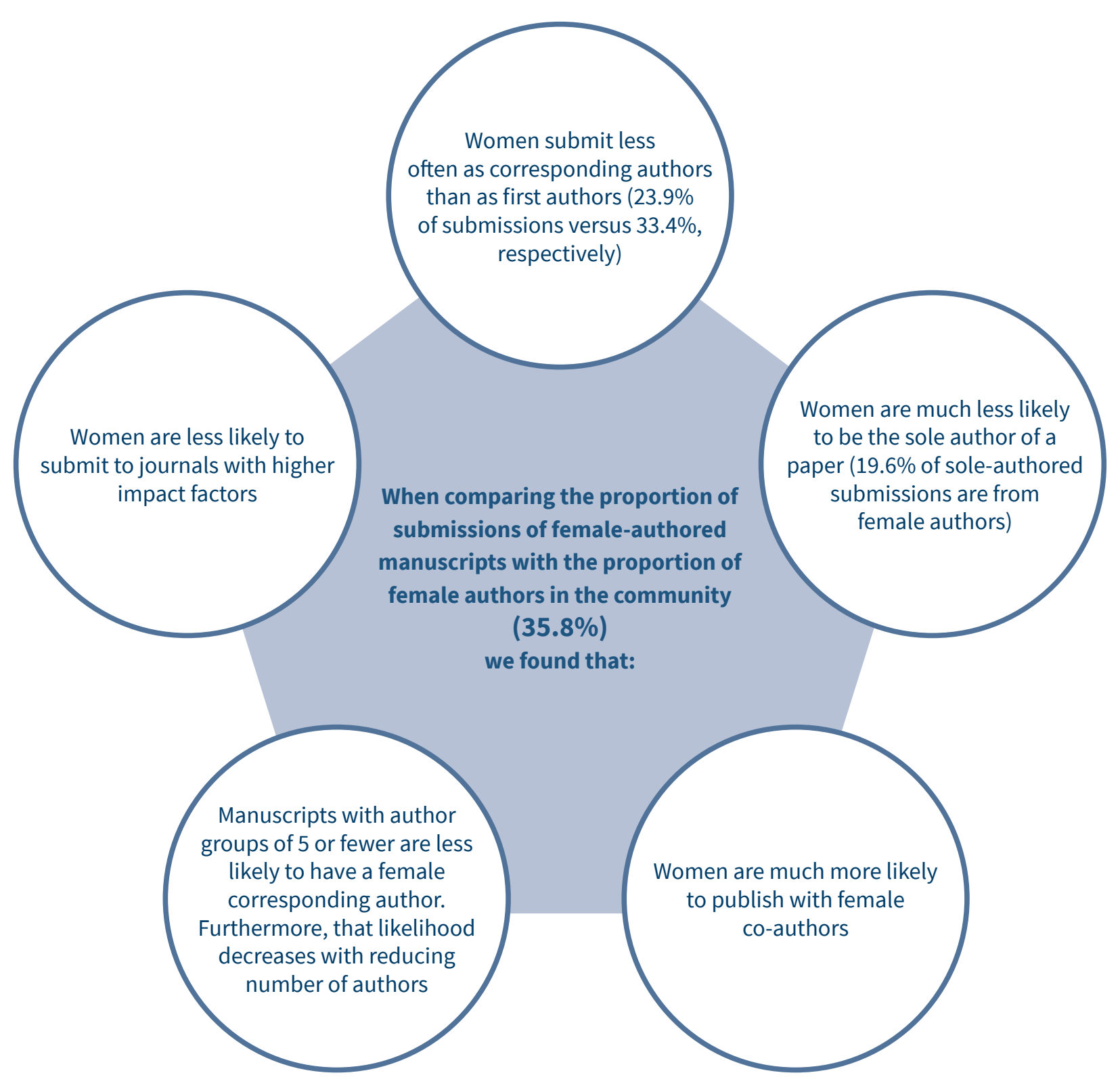


“/ am aware, based on personal experience and in my role as a Head of Department, that in general, it seems to be harder for female scientists to publish in a higher impact journal. It seems to me that these papers have to go through things [that] appear harsher. I think there is a direct correlation between [women being less likely to submit to high impact journals] and the difficulty in accepting a rejection."

Author and reviewer

“There are always exceptions, but I think women are much more careful about looking at the criteria. Men will say, 'my paper doesn't meet those 100\% but I'll go for it anyway.' Whereas women tend to say: 'I don't meet that particular criterion, I should therefore moderate which journal I go for.' I think that may well be a factor."

Author, reviewer and guest editor
6/ see [this] with my students. I will say, look at the highest impact journal or the place where you think your paper is the best suited and you would love to be able to publish in, and my female students will systematically say 'that's not going to get through'. Whereas, in general, I think my male students are more likely to just go for it." Author and reviewer

"6/ see no valid reason why women shouldn't be submitting [...] to highquality journals. In my experience there is no variation in the quality of the science."

Author, reviewer and editor

\section{They also indicate that time pressures and balancing professional and personal responsibilities} may play a role:

"..because of reduced time, for high level academics there are certain incompatibilities with family responsibilities. You start to think 'can I really afford to get this paper rejected twice at different journals? No, I'm just going to go for a safe bet because I need to publish this.' This is not something that is applicable only to females, I've seen male colleagues who've got stronger family commitments or caring responsibilities struggling. The current system is self-supporting and self-promoting a very nondiverse set of authors."

Author and reviewer

7.

\section{How does gender affect manuscript review and acceptance rates?}


The academic publishing process relies on the ability of editors, reviewers and other stakeholders, such as editorial boards, to act as gatekeepers in order to uphold the quality and novelty of research publications. To determine whether gender biases exist during this process, we looked at the gender profile at each stage of review, from initial assessment of the article based on suitability for the journal, to the final editorial decision to accept, revise or reject a manuscript.

In terms of accepted manuscripts, we found a slightly lower percentage by female corresponding authors and female first authors compared to those originally submitted.

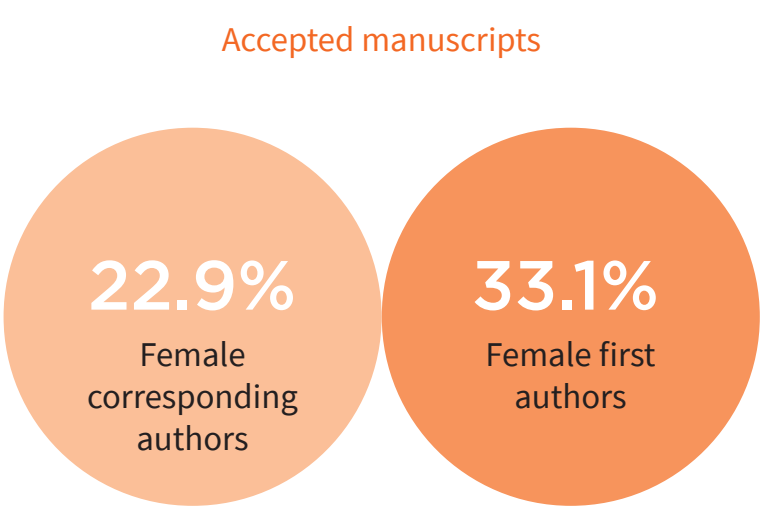

Original submitted manuscripts

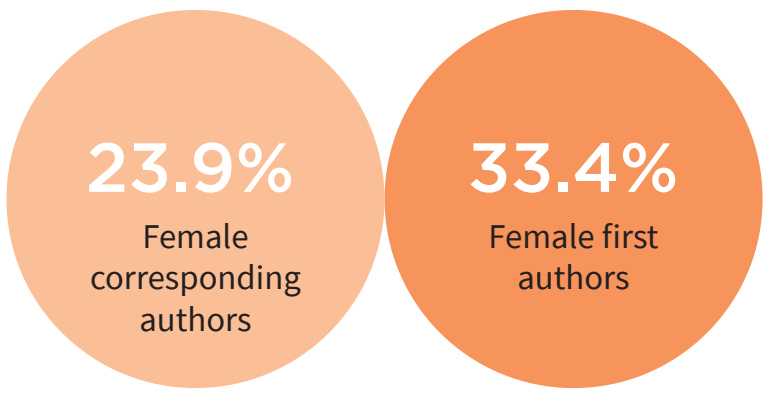

This aligns with the IOP's findings, which stated that This a ligns female corresponding authors have a slightly lower chance of being accepted ${ }^{3}$.

Looking more closely at the data for our journals indicates the existence of biases at two review stages: hitial assessment by the editor and peer review.

Regarding the first point, we found that initial submissions from female corresponding and first authors are more likely to be rejected without

peer review compared to the average percentage of all research manuscripts (rejected and accepted submitted by female corresponding authors and female first authors.

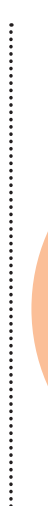

Rejected without peer review

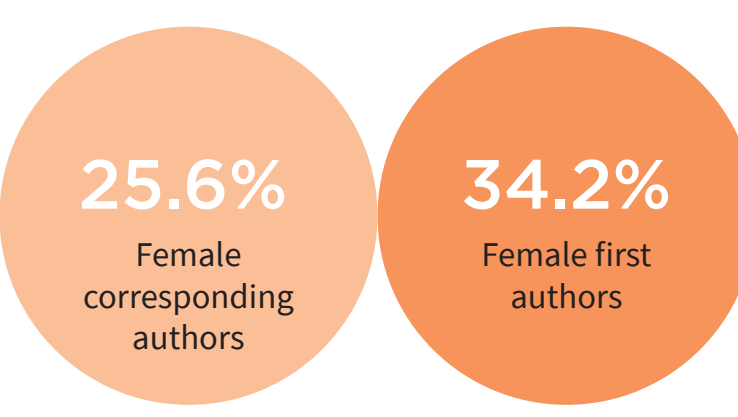

All research manuscripts (rejected and accepted)

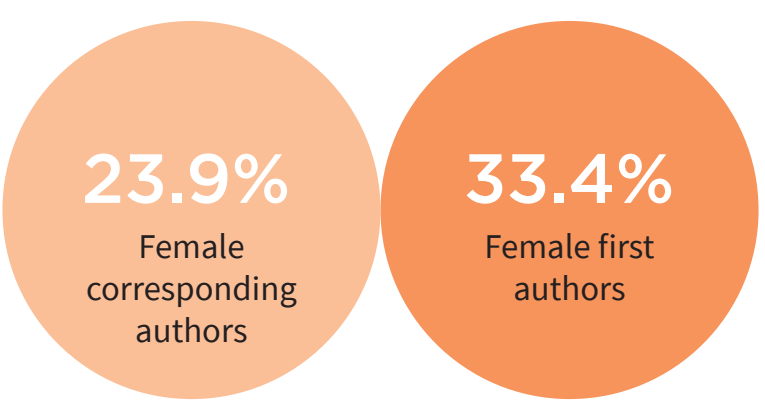

Our interviewees considered manuscripts being rejected without peer review to be a considerable setback for women (and potentially other minority groups) and a missed opportunity. Receiving constructive feedback would better support their progression:

"One of the biggest problems based on my experience is not the actual reviewing but the screening of the editors. The key process is in passing the editorial [review stage]. What are the key parameters that editors use that decide whether to reject a paper at the initial screening stage? Because really there is no indication and no feedback on that process. That is what is turning off an enormous amount of people."

Author and reviewer

Later in the publishing pipeline, biases operate in both positive and negative ways towards both men and women. We found that:

More manuscripts from male corresponding authors are recommended by reviewers to be accepted or to require minor revisions than those from women

Overall, reviewers are more likely to recommend rejection or major revisions, rather than

acceptance or minor revisions, for submissions from female corresponding authors than from male corresponding authors

Female reviewers are more likely to recommend major revisions rather than rejection

Female reviewers accept or recommend minor revisions for submissions from female corresponding authors more than their male counterparts

Male reviewers recommend rejection for submissions from female corresponding authors more than male corresponding authors 
These trends agree with the analysis of elife's article submissions ${ }^{6}$, which showed that both editors and reviewers favour manuscripts from authors of the same gender and same country.

"I / have never, ever looked at a gender of a corresponding author. However, I think there is an unconscious bias when you know the author, and so potentially this could be a gender bias. $"$

Author and reviewer

"Sometimes editors are biased... but I never had any reason to believe that it was because of my gender."

Author and reviewer
8.

\section{Are female authors cited less?}

The impact of biased reviews might be even more profound if the researcher is already working hard to overcome existing barriers to progression:

"I know there's all kind of stuff out there about trying to mentor women but the mentoring isn't going to do them any good if they get reviewed by biased reviewers. All the mentoring in the world isn't going to change that. In fact, it's only going to make things worse because they're going to think they're still not doing something right when it's really not their fault."

Author, reviewer and contributing editor

We also found that there is gender bias at the editorial decision-making stage, suggesting a propensity for female editors to choose and agree

Female editors are more likely to choose

female reviewers.

- Male editors are more likely to choose female reviewers for articles by female corresponding authors.

- Editors are more likely to agree with female reviewers especially if the editors are themselves women.

This was perceived by researchers we spoke to as a positive proactive bias of women being mo favourable towards women. Factors outside of the scope of this report may be at work.
6/ do recognise editors are under enormous pressure... the sheer number of submissions is so overwhelming."

Author and reviewe

6) think the executive editors do the best they can to try to identify women and also to get other sorts of diversity as well into our boards." Author and reviewer 
Papers by female corresponding authors are cited less than those from male corresponding authors (papers by female corresponding authors corresponding authors are cited 72 times)

- Men cite female corresponding authors less than male corresponding authors $17.8 \%$ of citations from male corresponding authors are to female corresponding authors)
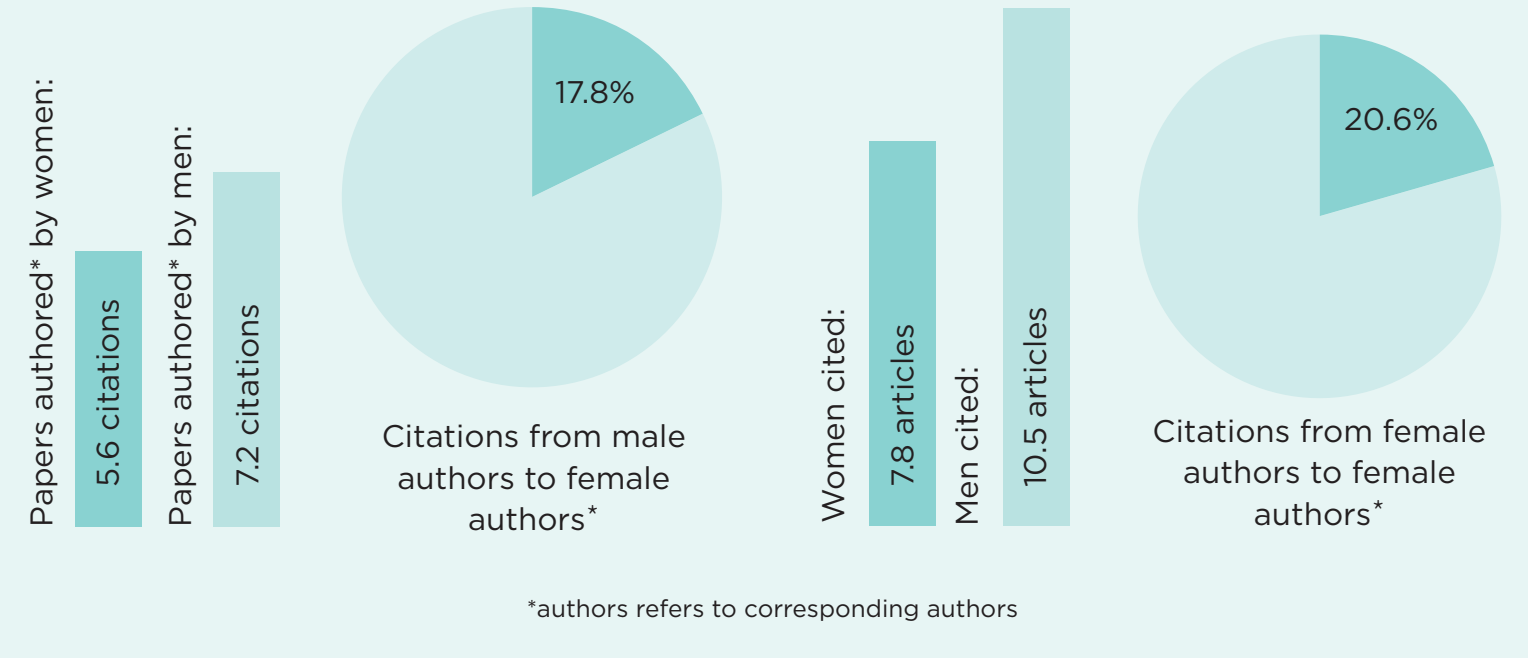

These results were mirrored by the experiences of some interviewees:

"I've experienced times where my papers have not been cited. I reviewed a book where there are two chapters devoted to research in which l've published seminal papers. There are about 300 references in there and I'm not cited at all. I didn't realise how important this was at the time.?

Author, reviewer and edito

"I know somebody who has a first name that is [] male-sounding and also someone who travels to a lot of meetings and gets cited a lot." "

Author, reviewer and contributing editor
Women cite fewer articles overall (on average

Women corresponding authors cite papers by authors are to female corresponding authors) female corresponding authors more than men do (20.6\% of citations from female corresponding
:6 think I suffer from this. I have papers that have been published in very good journals, but don't get the citation that you would expect.... genuinely believe that citations are about networks." Author, reviewer and editor
6) do think it's quite common that there is a laziness or a very obvious bias. You reference the people that you know, or who you're most familiar with: 'I must reference that person because I saw them at a conference or I saw them give a talk, or I saw them on a panel.' It's who you interact with. I suspect if you're a male researcher who interacts very strongly with female colleagues and female collaborators and has that wider network then the natural tendency will be to cite their work, but I can see how that bias might arise. 9

Author, reviewer and guest editor
"Citation is sometimes a really quick job. The reality is that probably 90\% of citations are carried out by students, and in a paper with 30 citations, there might be five or six that I feel strongly about based on scientific criteria and I say to the student make sure you include these papers. When I'm picking up references using keywords, nobody ever looks at the gender. I think it's the result of the smaller numbers [of women]. If I take any topic and I turn on Google Scholar. probably only two out of the first ten references are female. I think it's a selfperpetuating thing." Author, reviewer and guest editor

Taken together, these results suggest that even when papers authored by women are published, their work is less likely to be cited. However, we cannot be sure whether this is due to a true gender bias, the result of hasty citation practices, or simply the smaller proportion of female authors available to cite. Although women tend to cite other women, there are fewer female authors in the researcher pool to do so.

Whatever the cause, the overall result is that women are significantly less prominent in the scientific literature, and less visible to those looking to commission articles or invite peer reviewers-and the cycle perpetuates. 
Our analyses of gender bias across the Royal Society of Chemistry's publishing pipeline has highlighted several areas of imbalance in representation of male and female researchers in the chemical sciences.

This imbalance begins with the allocation of position in the author list and the choice of where to submit

a research paper. We then found that a higher

proportion of women had their articles rejected

after initial assessment. Although authors may

have received constructive input on the suitability of

their manuscript for the journal from the editor, for

many authors this results in a missed opportunity to receive a detailed critique from several scholars in their field.

Our data and our interviews with researchers in our

community suggest that the imbalance is persisting in failing to provide an unbiased constructive critique of

How do we tackle these biases within the publishing process?

We asked members of our publishing community what they thought needed to change based on their experiences and the data presented here. A number of common themes emerged from these discussions:

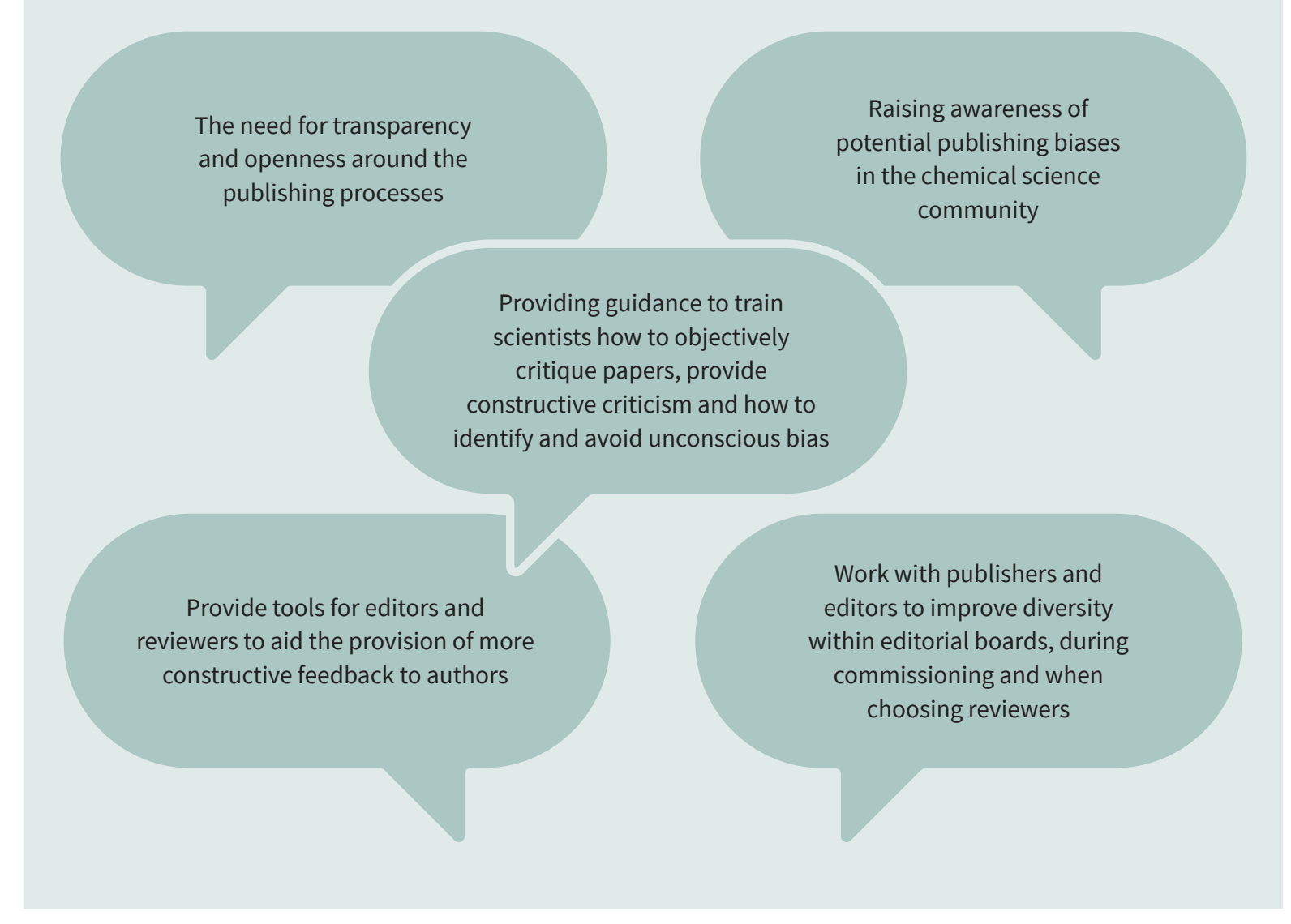


"The first step I think is awareness that there is bias here. The numbers show it, so the first part is acknowledging it. I don't think it's as simple as just saying that we have equality in the balance of the reviewers. That's going to be very difficult to achieve and it could backfire, because you end up asking your female colleagues to do more reviewing or sitting on appointment panels rather than actually doing their next grant application or writing their next paper. But it's about having that awareness and that scrutiny of the reviewers to see, well, is there systematic bias in particular reviewers? I think it's something that the chemistry community needs to take hold of and own."

Author, reviewer and editor
"There is certainly a lack of diversity and I think this lack of diversity has to be addressed. I don't think it is all on the side of the publishing houses, there has to be support within the community, and from people like myself in leadership roles."

Author, reviewer and editor

"There has to be more transparency within the community about the challenges we all face." Author, reviewer and editor
10.

\section{What we will do}


As part of our strategy on inclusion and diversity, we are committed to continuing to scrutinise our own publishing processes, in order to address gender bias at each stage of ine publishing pipeline. This activities. We aim to increase awareness of these nd impacted by, our publishing processes.

\section{We have identified four key areas for action. We will:}

\section{Increase transparency}

Undertake comprehensive analysis and reporting of our authors, reviewers and ditorial decision makers by sub-discipline - and publish this annually. We call on is to do the same.

2 Reflect our research community

Recruit and train reviewers, editorial board members and associate editors to reflect the current gender balance of our research community: our target for 2022 is at least $36 \%$ women.

3 Empower and innovate

Provide new training and resources to empower our editors to eliminate bias. We will test new models throughout the publishing profile to address bias from submission to publication.

4 Encourage intervention

Partner with others and lead the development of a new Inclusion \& Diversity Framework for Action to set the standard for driving change within the academic publishing industry.

Our call to other publishers

We call on other scientific publishers to commit to the same scrutiny of their own processes - to join us in reporting on their own activities.

Our call to the research community

We call for the chemical science community to be aware of the biases identified here. We must all consider how we recognise, name and avoid biases in the future. Ensuring that our publications feature a more diverse range f voices will lead to better science, a more diverse and productive chemistry workforce for the future ${ }^{3}$, and will benefit all in society.

"Our challenge to publishers, editors and referees alike is to do more to check at every stage that there is no lurking bias, implicit or explicit - and to think about the knock-on effects, for gender equality, of everything they do."

Melinda Duer and Dame Athene Donald Times Higher Education supplement 2019

11.

\section{References and acknowledgments}


1. The Royal Society of Chemistry, 2018. Diversity landscape of the chemical sciences. Available:

www.rsc.org/globalassets/02-about-us/our-strategy/inclusion-diversity/cm-044-17_a4-diversitylandscape-of-the-chemical-sciences-report_web-2.pdf

2. The Royal Society of Chemistry, 2018. Breaking the Barriers: Women's retention and progression in the chemical sciences. Available: www.rsc.org/globalassets/02-about-us/our-strategy/inclusion-diversity/ womens-progression/media-pack/v18_vo_inclusion-and-diversity-_womans-progression_reportweb-.pdf

3. Freeman and Huang, Journal of Labor Economics 2015, 33, S289

4. Elsevier, 2017. Gender in the Global Research Landscape. Available:

www.elsevier.com/__data/assets/pdf_file/0008/265661/ElsevierGenderReport_final_for-web.pdf

5. The Institute of Physics, 2018. Diversity and Inclusion in Peer Review at IOP Publishing.

Available: www.ioppublishing.org/wp-content/uploads/2018/09/J-VAR-BK-0818-PRW-report-final2.pdf

6. Murray D et al, 2018. Gender and international diversity improves equity in peer review. Available: www.biorxiv.org/content/biorxiv/early/2018/08/29/400515.full.pd

7. UK Intellectual Property Office, 2016. Gender Profiles in UK Patenting. Available:

www.gov.uk/government/publications/gender-profiles-in-uk-patenting-an-analysis-of-femaleinventorship

8. Is there a gender gap in chemical sciences scholarly communications? Day, Corbett and Boyle, available from ChemRXiv, Under Review

Acknowledgments:

This work, led by the Royal Society of Chemistry Inclusion and Diversity team, follows on from our latest report Breaking the Barriers and is part of an on-going collaborative project with the Publishing team.

We thank individuals who have taken part in interviews and those who provided input throughout the analysis and development of the report, in particular we thank members of the Royal Society of Chemistry Inclusion and Diversity Committee and the Publications Board.

We also thank the Royal Society of Chemistry Data Science team for the data analysis and contributions. 


\section{ROYAL SOCIETY OF CHEMISTRY}

Thomas Graham House

Science Park, Milton Road

Cambridge CB4 OWF, UK

$\mathrm{T}+44(0) 1223420066$

Burlington House

Piccadilly, London

W1J OBA, UK

$\mathrm{T}+44(0) 2074378656$

International offices

Beijing, China

Shanghai, China

Berlin, Germany

Bangalore, India

Tokyo, Japan

Philadelphia, USA

Washington, USA

WwW.rsc.org

f @RoyalSocietyofChemistry

y@RoySocChem

○ @roysocchem

D@wwwRSCorg

in linkedin.com/company/roysocchem 